\title{
Corrigendum
}

Mathilde Bonnet*, Marine Dermu, Clara Roessle, Marc Bellaiche, Tarik Abarou, Véronique Vasseur, Samira Benakouche and Thomas Storme

\section{Corrigendum to: Formulation of a 3-months Stability Oral Viscous Budesonide Gel and Development of an Indicating Stability HPLC Method}

https://doi.org/10.1515/pthp-2018-9005

Corrigendum to: Mathilde Bonnet, Marine Dermu, Clara Roessle, Marc Bellaiche, Tarik Abarou, Véronique Vasseur, Samira Benakouche and Thomas Storme. Formulation of a 3-months Stability Oral Viscous Budesonide Gel and Development of an Indicating Stability HPLC Method. Pharmaceutical Technology in Hospital Pharmacy. Volume 3, Issue 2, pages 91-99. (DOI https://doi.org/10.1515/pthp2018-0005)
*Corresponding author: Mathilde Bonnet, Pharmacy, Hopital Universitaire Robert-Debre, 48 boulevard Sérurier, Paris 75019, France, E-mail: mathildb@hotmail.fr

Marine Dermu, Pharmacy, Hopital Universitaire Robert-Debre, 48 boulevard Sérurier, Paris 75019, France

Clara Roessle, Pharmacy, Hopital Universitaire Robert-Debre, 48 boulevard Sérurier, Paris 75019, France

Marc Bellaiche, Gastroenterology, Hopital Universitaire RobertDebre, Paris, Île-de-France, France

Tarik Abarou, Véronique Vasseur, Samira Benakouche, Thomas

Storme, Pharmacy, Hopital Universitaire Robert-Debre, 48 boulevard

Sérurier, Paris 75019, France
In Table 1 , on page 94, the formulation was based on the original formula published by Eyal Zur, compounding pharmacist and consultant from Israel, in the International Journal of Pharmaceutical Coupounding in 2012. [1]

[1] Zur, E. 2012. Eosinophilic esophagitis: treatment with oral viscous budesonide. Int. J. Pharm. Compd. 16: 288-293. 